Open Access

\title{
The HDL receptor SR-BI is associated with human prostate cancer progression and plays a possible role in establishing androgen independence
}

\author{
David Schörghofer ${ }^{1 \dagger}$, Katharina Kinslechner ${ }^{1 \dagger}$, Andrea Preitschopf ${ }^{1}$, Birgit Schütz ${ }^{1}$, Clemens Röhrl $^{2}$, \\ Markus Hengstschläger ${ }^{1}$, Herbert Stangl ${ }^{2}$ and Mario Mikula ${ }^{1 *}$
}

\begin{abstract}
Background: Human prostate cancer represents one of the most frequently diagnosed cancers in men worldwide. Currently, diagnostic methods are insufficient to identify patients at risk for aggressive prostate cancer, which is essential for early treatment. Recent data indicate that elevated cholesterol levels in the plasma are a prerequisite for the progression of prostate cancer. Here, we analyzed clinical prostate cancer samples for the expression of receptors involved in cellular cholesterol uptake.
\end{abstract}

Methods: We screened mRNA microarray files of prostate cancer samples for alterations in the expression levels of cholesterol transporters. Furthermore, we performed immunohistochemistry analysis on human primary prostate cancer tissue sections derived from patients to investigate the correlation of SR-BI with clinicopathological parameters and the mTOR target pS6.

Results: In contrast to LDLR, we identified SR-BI mRNA and protein expression to be induced in high Gleason grade primary prostate cancers. Histologic analysis of prostate biopsies revealed that $53.6 \%$ of all cancer samples and none of the non-cancer samples showed high SR-BI staining intensity. The disease-free survival time was reduced $(P=0.02)$ in patients expressing high intra-tumor levels of SR-BI. SR-BI mRNA correlated with HSD17B1 and HSD3B1 and SR-BI protein staining showed correlation with active ribosomal protein $\mathrm{S} 6$ (RS $=0.828, P<0.00001$ ).

Conclusions: We identified SR-BI to indicate human prostate cancer formation, suggesting that increased levels of SR-BI may be involved in the generation of a castration-resistant phenotype.

Keywords: SCARB1, LDLR, Cholesterol, mTOR, Androgen synthesis

\section{Background}

Prostate cancer is one of the most common solid organ tumors in males. It is a slow growing type of tumor, but can potentially give rise to aggressive and metastasizing forms of cancer [1]. The risk for prostate cancer increases with consumption of a high fat, high cholesterol diet or the presence of hypercholesterolemia [2-4]. Very recently, it was shown that the accumulation of esterified cholesterol underlies the aggressiveness of human prostate cancer [5]. Cellular cholesterol is either synthesized by the

\footnotetext{
*Correspondence: mario.mikula@meduniwien.ac.at

'Equal contributors

${ }^{1}$ Institute of Medical Genetics, Medical University of Vienna, Währinger

Strasse 10, 1090 Vienna, Austria

Full list of author information is available at the end of the article
}

cells themselves, or exogenous cholesterol is taken up and utilized by the cancer cells. Cholesterol uptake is mainly mediated by the high density lipoprotein receptor SR-BI and the low density lipoprotein receptor LDLR [6-9]. In normal tissue, SR-BI is expressed in the liver and in steroidogenic tissues, where cholesterol uptake is necessary for steroid hormone synthesis [10-13]. Notably, patients suffering from mutations in cla-1, the human homolog to SR-BI, display impaired steroid hormone synthesis [14]. There is evidence that SR-BI plays a role in prostate cancer development, specific antigen secretion and the viability of prostate cancer cells because it was shown that SR-BI-specific knockdown in LNCaP and C4-2 prostate carcinoma cells reduced PSA secretion and the viability of 
prostate cancer cell lines [15]. Therefore, this study aimed to evaluate the expression of receptors involved in cellular cholesterol uptake in clinical prostate cancer samples.

\section{Material and methods Bioinformatic analysis}

For Gleason score analysis, the GSE2109 and GSE3933 datasets from the International Genomics Consortium Expression Project for Oncology were used [16]. The sample sizes were as follows: GSE2109, $n=56$ (Gleason score $\leq$ $6 n=20$, Gleason score $\geq 7 n=36$ ), GSE3933, $n=58$ for SR-BI (Gleason score $\leq 6 n=24$, Gleason score $\geq 7 n=34$ ) and $n=60$ for LDLR (Gleason score $\leq 6 n=24$, Gleason score $\geq 7 \mathrm{n}=36$ ). For metastasis analysis, the GSE35988, GSE3933 and GSE6919 datasets were used [16-19]. The sample sizes were as follows: GSE35988, $n=94$ (primary site $n=59$, metastasis $n=35$ ), GSE3933, $n=68$ for SR-BI (primary site $n=59$, metastasis $n=9$ ) and $n=68$ for LDLR (primary site $n=61$, metastasis $n=7$ ) and GSE6919, $n=88$ (primary site $n=64$, metastasis $n=24$ ). For Kaplan-Meier analysis, the GSE40272 dataset was used (sample size: $n=85)$ [20].

\section{Patient cohort and pathology}

With institutional review board approval from the Medical University of Vienna (EK Nr: 1734/2014), tissue microarrays were obtained from US Biomax (Rockville, MD). All samples were formalin-fixed less than $10 \mathrm{~min}$ after surgery, paraffin embedded and assembled as cores with a diameter of $1.5 \mathrm{~mm}$. Tissue sections were quality controlled and contained normal prostate tissue and prostate cancer tissue, representing different stages of disease progression. Each individual core was assigned to independent Gleason scoring and was reviewed by two boardcertified pathologists.

\section{Immunohistochemistry}

Prostate cancer tissue sections containing paraffinembedded samples were melted for $20 \mathrm{~min}$ at $60{ }^{\circ} \mathrm{C}$ and rehydrated by subsequent incubation in Xylol, Isopropanol, 96 \% Ethanol, 70 \% Ethanol and $50 \%$ Ethanol. Then, tissue sections were washed and heated to $120{ }^{\circ} \mathrm{C}$ in a pH 6.0 buffer or a pH 9.0 buffer (Dako, Glostrup, Denmark), depending on the antibody. After cooling to room temperature, samples were incubated with $1 \% \mathrm{H}_{2} \mathrm{O}_{2}$ (Sigma, St. Louis, MO) for $10 \mathrm{~min}$. Afterwards, samples were permeabilized with $0.1 \%$ Triton X-100 (Sigma) for $5 \mathrm{~min}$. Then, sections were blocked with $2.5 \%$ horse sera (Vector Laboratories, Burlingame, CA) for at least $20 \mathrm{~min}$ at room temperature. Subsequently, sections were incubated overnight at $4{ }^{\circ} \mathrm{C}$ with the primary antibodies directed against SR-BI (BD Transduction Laboratories ${ }^{\mathrm{Tm}}$, Franklin Lakes, NJ),
LDLR (Santa Cruz Biotechnology, Santa Cruz, CA) and pS6 (Cell Signaling Technology, Beverly, MA), diluted 1:200. For negative control staining, sections were incubated with matched isotope control antibodies instead of primary antibodies. Next, slides were washed and the corresponding secondary, biotinylated antibodies (Vector Laboratories) were added for $45 \mathrm{~min}$ at room temperature. After a washing step, sections were incubated for $30 \mathrm{~min}$ with Streptavidin-HRP (Leica, Wetzlar, Germany). For detection, tissue sections were incubated with AEC+ High Sensitivity Substrate Chromogen (Dako). Counterstaining with hematoxylin solution was performed according to Mayer (Carl Roth, Karlsruhe, Germany); tissue sections were mounted with Aquatex ${ }^{\odot}$ (Merck Millipore, Billerica, MA).

\section{Evaluation of immunohistochemical staining}

Evaluation of tissue sections was performed by two independent researchers who were blinded to the patients' details. Immunostaining of the anti-SR-BI antibody was scored on at least duplicate tissues using the following arbitrary scale: no staining (0), low staining (1), medium staining (2) and high staining (3).

\section{Statistical analysis}

Dot plots were generated with SPSS v21. The arithmetic mean of all samples is indicated by a line. Two-tailed $P$ values were calculated with unpaired (independent) t-tests in SPSS. $P$-values $\leq 0.05$ were considered to be statistically significant. The significance of the difference between the variances of two samples was determined with Levene's test. If the resulting P-value of Levene's test was $>0.05$, we assumed equal variances and adopted the output of the equal variance $t$-test as the $P$-value; if the resulting P-value of Levene's test was $\leq 0.05$, we assumed unequal variances and adopted the output of the unequal variance $t$-test as the $P$-value.

Scatter plots were generated with SPSS v21. Pearson correlation analysis was performed to calculate the P-values of the graphs.

For analysis of immunohistological staining results, the internet tool VassarStats (http://vassarstats.net/index.html) was used. Risk ratios were calculated using $2 \times 2$ contingency tables and the chi-square test was applied to determine the association of clinicopathological parameters with SR-BI expression.

Kaplan-Meier analysis and the log-rank test were performed using SPSS 21 to test the association of SRBI and LDLR with disease-free survival time. A total of 85 samples were available for evaluation. For the analysis, samples were segregated into groups with SRBI or LDLR levels above (42 samples) and below or equal (43 samples) to the median value. 


\section{Results}

Evaluation of SR-BI and LDLR expression as markers for prostate cancer progression

We analyzed SR-BI and LDLR mRNA expression in primary human prostate cancers from the Expression Project for Oncology and from a hallmark study by
Lapointe et al. [16]. When comparing prostate cancer samples with high Gleason scores (equal to or higher than 7) and samples with low Gleason scores (equal to or lower than 6), SR-BI was more highly expressed in GSE2109 $(n=56, P=0.039$, Fig. $1 \mathrm{a})$ and GSE3933 $(n=58, P=0.016$, Fig. $1 c)$. LDLR was not increased in
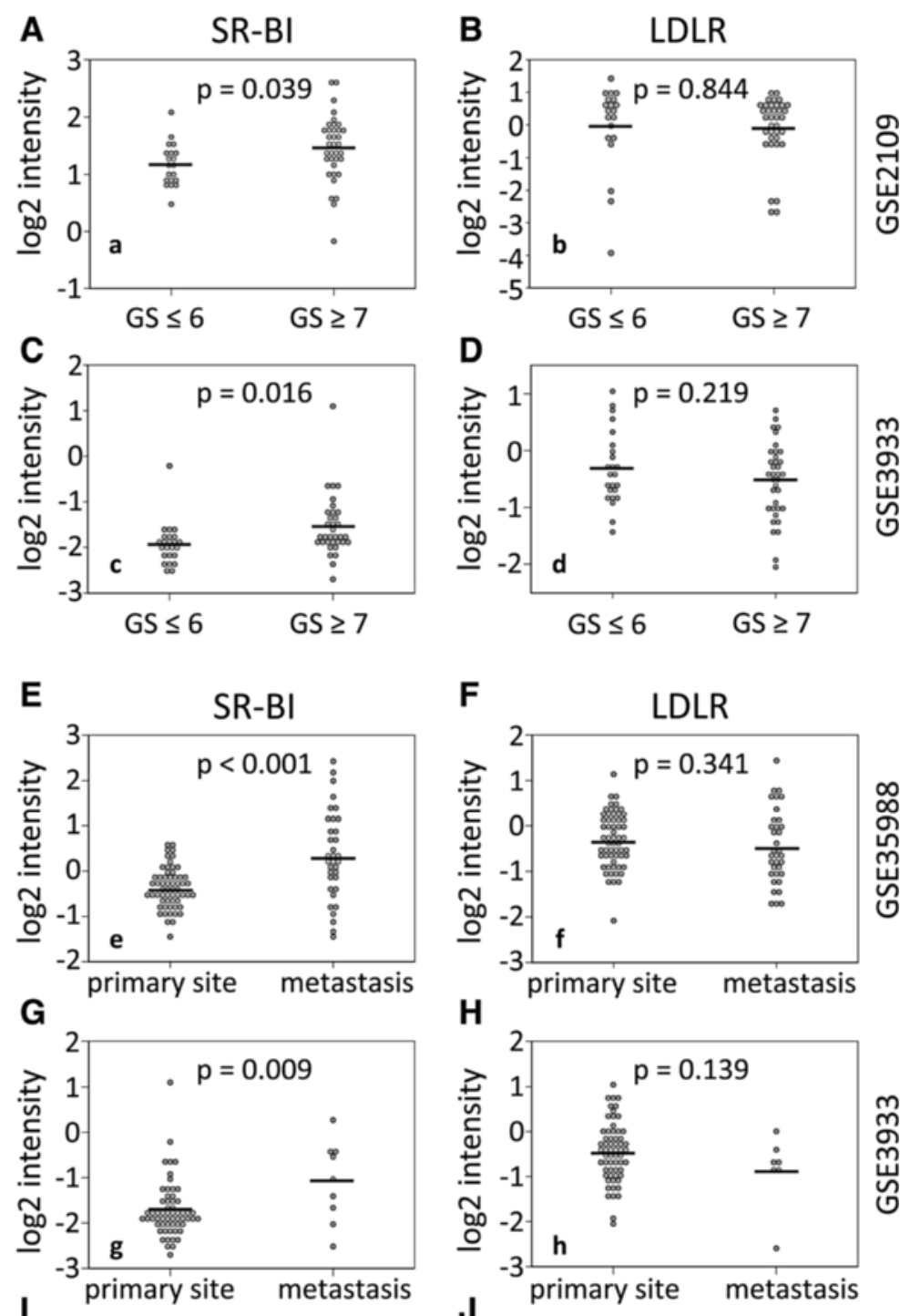

H
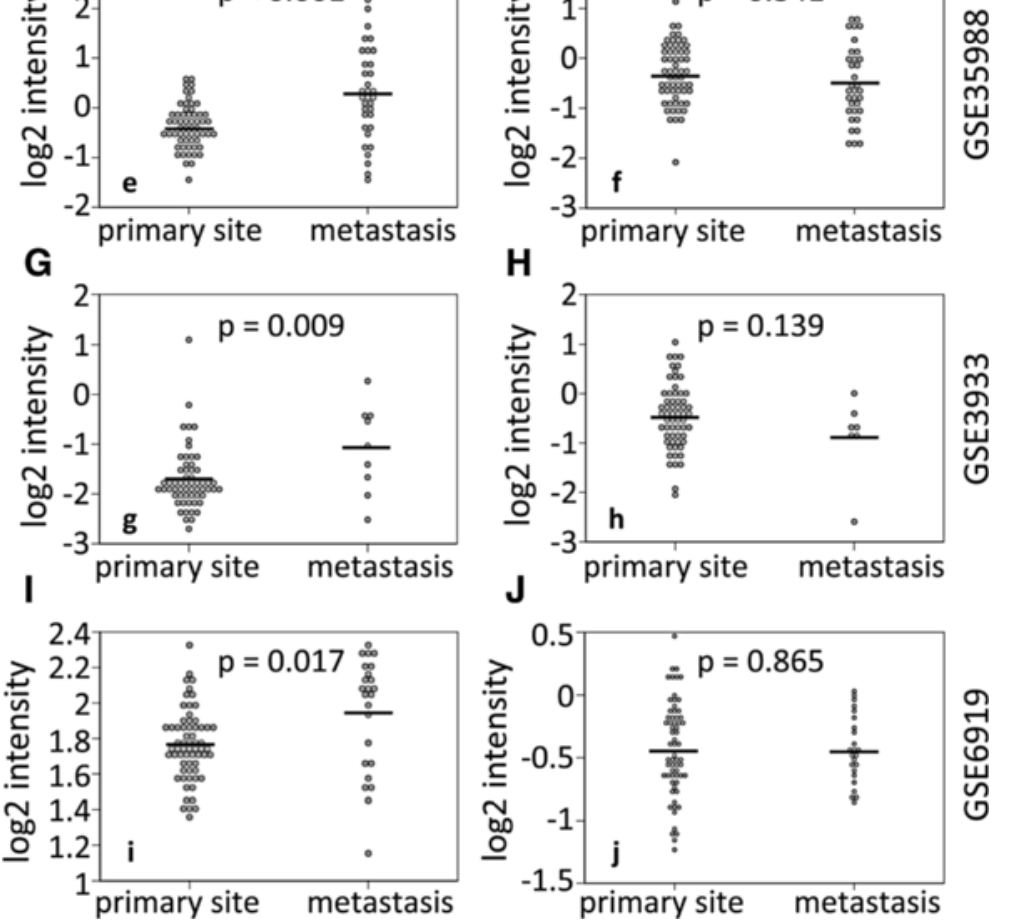

Fig. 1 Expression of SR-BI and LDLR in clinical prostate samples. Differential expression of SR-BI and LDLR according to Gleason scoring (a-d). All samples were grouped according to Gleason score (GS) $\leq 6$ and $\geq 7$. Differential expression of SR-BI and LDLR in metastasizing and non-metastasizing prostate tissues (e-j). All samples were grouped into primary tumors (primary site) and metastasizing tumors (metastasis). P-values are presented within each graph. The arithmetic mean is given as a line within the dots. Data displayed as log 2 median-centered intensity 
high Gleason score samples in GSE2109 $(n=56, P=0.844$, Fig. 1b) and in GSE3933 ( $n=60, P=0.219$, Fig. $1 \mathrm{~d})$.

The most important parameter used to decide upon patient survival is the occurrence of metastasis. Therefore, we next determined the expression of SR-BI and LDLR in clinical prostate samples derived either from non-metastatic or metastatic prostate cancer. For this analysis, we investigated the dataset GSE35988, which contains benign and localized prostate cancer from radical prostatectomy as well as metastatic, castrationresistant prostate cancer obtained from rapid autopsy [17]. Furthermore, we included the datasets GSE6919 and GSE3933, which contain samples from the primary tumor site as well as metastasized prostate cancer samples from the liver, lung, kidney, adrenal gland or lymph nodes $[16,18,19]$. We identified an increased expression of SR-BI in metastatic prostate samples compared to non-metastatic prostate samples in GSE35988, GSE3933 and GSE6919 $(P<0.001, P=0.009$ and $P=0.017$, respectively) (Fig. $1 \mathrm{e}, \mathrm{g}, \mathrm{i}$, ). Contrary to SR$\mathrm{BI}$, LDLR expression was not increased in metastatic prostate samples compared to non-metastatic prostate samples in GSE35988, GSE3933 and GSE6919 $(P=0.341, P=0.139$ and $P=0.856$, respectively) (Fig. 1f, h, j).

\section{Association of clinicopathological parameters with SR-BI expression}

To confirm findings from mRNA expression studies, we assessed SR-BI protein expression in normal prostate tissue and prostate cancer samples derived from patients with known TNM status. A total of 106 biopsy cores were independently assigned to Gleason scoring. Cores were subjected to immunohistochemical staining for SR-BI and afterwards analyzed for their staining intensity. Table 1 shows the clinicopathological characteristics of the cohort studied. Samples were classified into non-cancer and cancer samples. Cancer samples were further characterized by Gleason score, pathologically classified tumor stage (pT) and metastasis. Nearly half of all cancer samples

Table 1 Frequencies of clinicopathological characteristics

\begin{tabular}{lll}
\hline Characteristics & & Sample size \\
\hline Type & Non-cancer & 23 \\
& Cancer & 83 \\
Gleason score & $\leq 6$ & 38 \\
& $\geq 7$ & 45 \\
Tumor stage & pT2 & 54 \\
& pT3/pT4 & 29 \\
Metastasis & Negative & 45 \\
& Positive & 38 \\
\hline
\end{tabular}

showed a Gleason score equal to or above 7. Furthermore, about a third of all cancer samples showed advanced tumor stages (pT3/4), and nearly half of them were positive for metastasis. Representative cores for different staining intensities of SR-BI and their respective scores are shown in Fig. $2(\mathrm{a}-\mathrm{d})$. We tested the specificity of our antibodies by staining human liver sections (Fig. 2e and $\mathrm{f}$ ). The distribution of the results for the whole tissue collective is shown in Fig. 2g. On the basis of a binary classification system for low (score 0 and score 1 ) and high (score 2 and score 3) SR-BI staining intensities, we evaluated associations between SR-BI and the presence or absence of clinicopathological parameters. Notably, out of 23 normal prostate samples, none showed high staining results (score 2 and score 3), and among all cancer samples, approximately $53.6 \%$ showed high staining for SR-BI.

As shown in Table 2, we identified high SR-BI expression to be associated with the presence of prostate cancer when compared to non-cancer prostate tissue (risk ratio $=2.154$, $P<0.0001)$. Furthermore, we identified an association of high SR-BI score with a Gleason score equal to or higher than 7 (risk ratio $=2.907, P<0.0001$ ).

Because SR-BI expression showed an association with prostate cancer differentiation, we also tested for LDLR expression on selected sections with either low or high SR-BI staining intensity (Fig. 3a-d). Our case study showed that LDLR was constitutively expressed in prostate tissue, with lower expression levels in highgrade cancer samples. Interestingly, we also observed cases of low-grade prostate cancer, which displayed high SR-BI expression in a subpopulation of cells showing signs of tissue invasion (Fig. 3e and f). Cancer cells of this subpopulation either grew detached from the primary tumor, floating in the remaining glands (Fig. 3e), or separated from the solid tumor mass, infiltrating the surrounding tissue (Fig. 3f).

To assess whether SR-BI and LDLR had any influence on the clinical outcome of patients, we chose to evaluate the disease-free survival time in relation to SR-BI and LDLR expression. Therefore, we performed KaplanMeier analyses of the dataset GSE40272, which contained mRNA expression data on prostate tissue samples from men who underwent radical prostatectomy [20]. The disease-free survival time was defined as the time between surgery and the recurrence of disease (serum PSA $>0.1 \mathrm{ng} / \mathrm{ml}$ on two consecutive measurements after surgery). We identified samples with low SR-BI expression to have a significantly better survival outcome compared to samples with high SR-BI expression $(\mathrm{p}=0.02$, Fig. 3h). By contrast, there was no significant difference in disease-free survival time between samples with low LDLR expression and samples with high LDLR expression (Fig. 3g). 

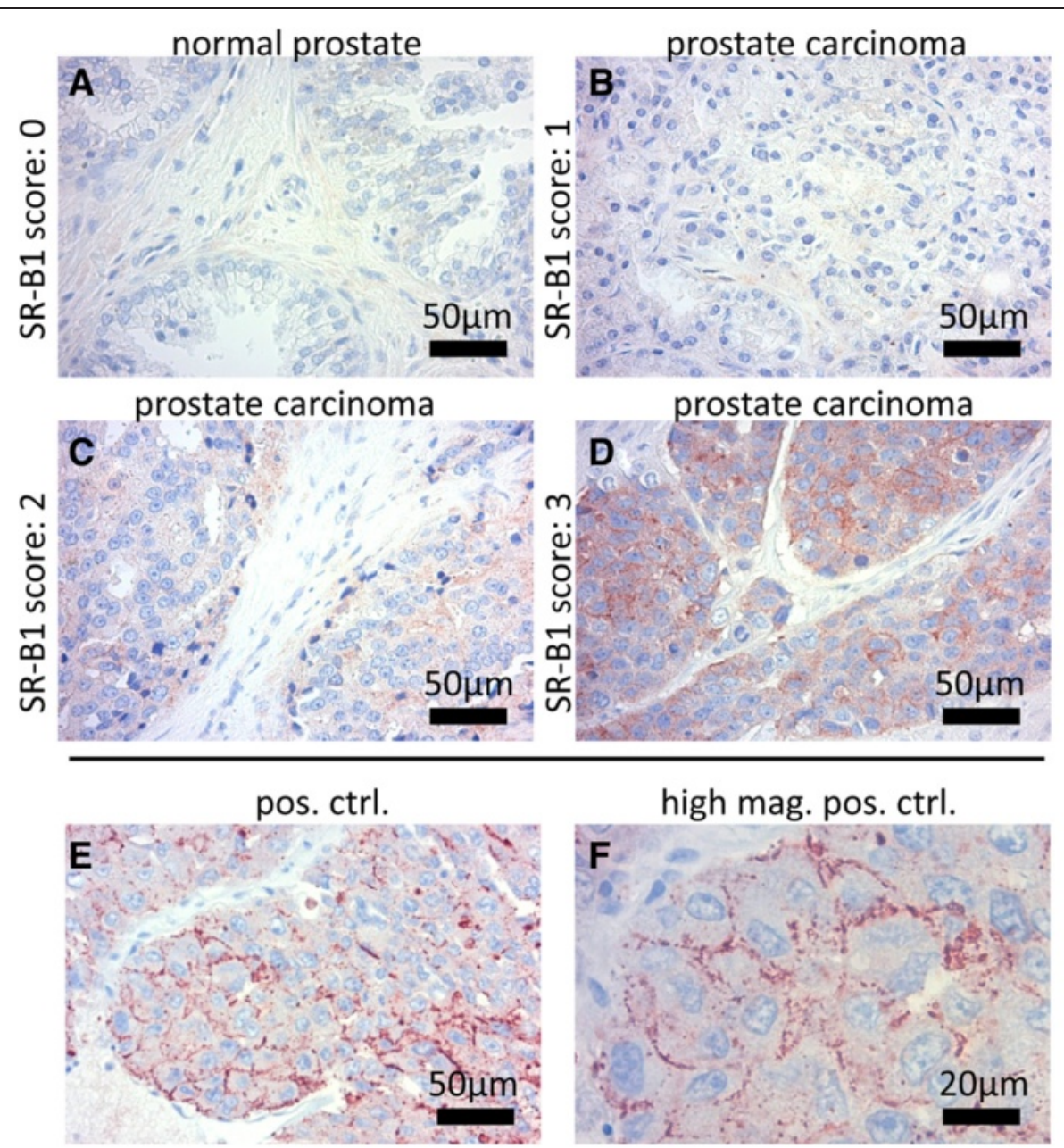

\section{G}

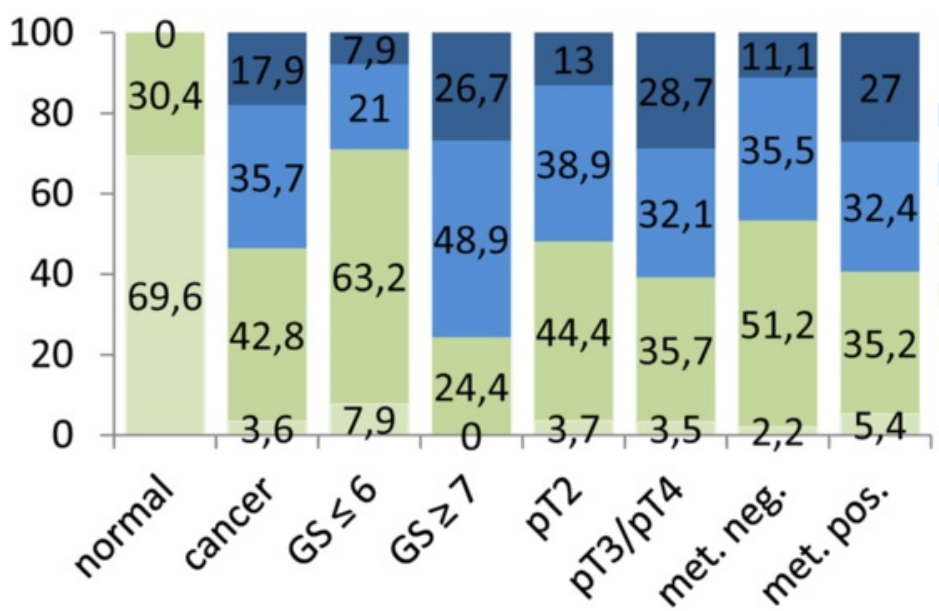

Fig. 2 Immunohistochemical staining of prostate tissue and control tissue for SR-BI expression. Each panel shows representative prostate samples scored for staining intensity as follows: 0 for negative (a), 1 for low (b), 2 for moderate (c) and 3 for high (d). Liver sections were used as positive controls (pos. ctrl.) in standard magnification (e) and in high magnification (high mag.), demonstrating SR-BI localization in the outer cell membrane of hepatocytes (f). The overall scoring distribution for SR-Bl staining intensity in different clinicopathological groups (g). Scores of 0 and 1 represented "low" expression and are shown in green colors, whereas scores of 2 and 3 represented "high" expression and are shown in blue colors. Clinicopathological groups are plotted on the x-axis: non-cancer and cancer, Gleason score (GS) $\leq 6$ and $\geq 7$, pathologic tumor stage 2 (pT2) and pathologic tumor stage 3/4 (pT3/4), metastasis negative (met. neg.) and metastasis positive (met. pos.) 
Table 2 Evaluation of the prognostic significance of SR-BI staining intensity

\begin{tabular}{llcl}
\hline Variable & $P$-value & Risk ratio & $95 \%$ Confidence interval \\
\hline Cancer vs. non-cancer & $<0.0001$ & 2.128 & $1.693-2.675$ \\
GS $\geq 7$ vs. GS $\leq 6$ & $<0.0001$ & 2.907 & $1.673-5.050$ \\
pT3/pT4 vs. pT2 & 0.371 & 1.269 & $0.739-2.182$ \\
Met. pos. vs. met. neg. & 0.207 & 1.351 & $0.837-2.182$ \\
\hline
\end{tabular}

Correlation of SR-BI with androgen-synthesizing enzymes and the mTOR pathway

Because SR-BI mediates the selective uptake of cholesterol, which can be used for steroidogenesis, we analyzed enzymes that participate in androgen synthesis. We identified the $\beta$-hydroxysteroid-dehydrogenases HSD17B1 and HSD17B3 to be significantly up-regulated in metastatic compared to non-metastatic prostate cancer; they also correlated with the intensity of SR-BI expression (Fig. 4a-h).

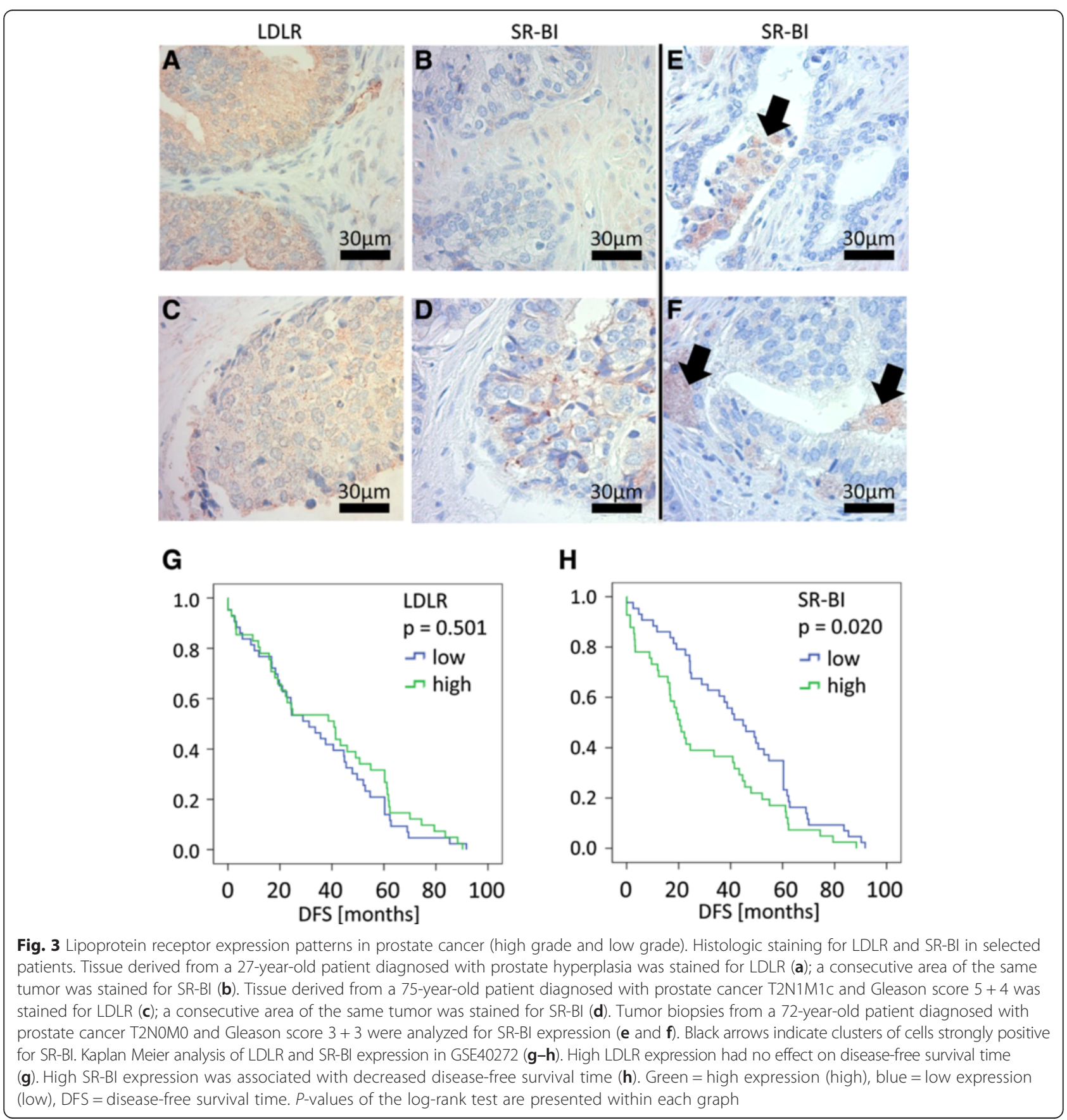




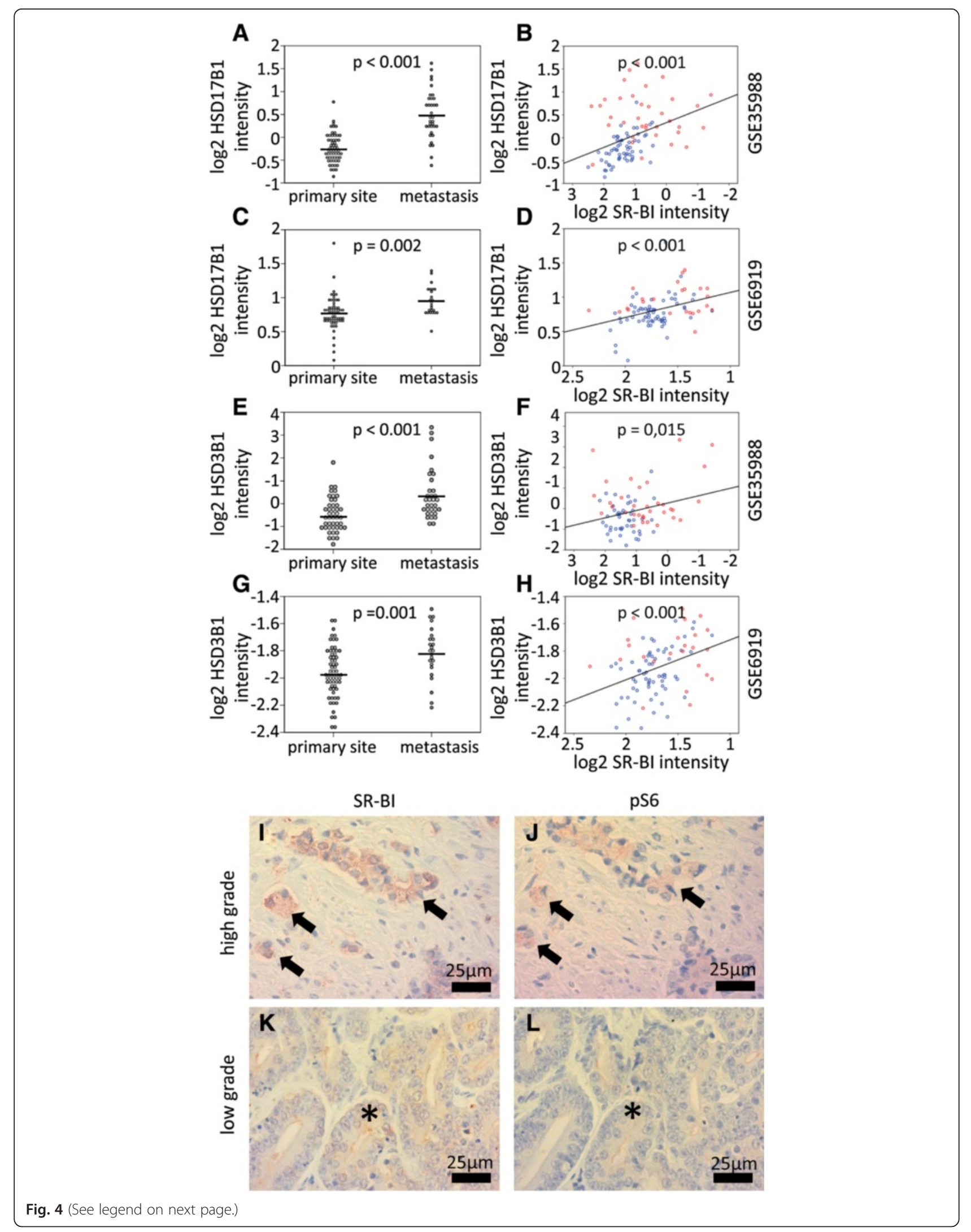


Fig. 4 Correlation of SR-BI with androgen-synthesizing enzymes and the mTOR pathway. Differential expression of HSD17B1 and HSD3B1 in primary tumors (primary site) and metastasizing tumors (metastasis) $(\mathbf{a}, \mathbf{c}, \mathbf{e}, \mathbf{g}$ ). The arithmetic mean is given as a line within the dots and the $P$-values of $t$-test analysis is given within each graph. Scatter plots correlating SR-Bl expression with HSD17B1 and HSD3B1 in prostate cancer (b, d, f, h). P-values of Pearson correlation analyses and regression lines are presented within each graph. Red dots represent metastatic and blue dots represent non-metastatic prostate cancer. Analysis of the co-occurrence of SR-BI and ribosomal protein S6 phosphorylation (i-I). A representative high grade prostate cancer is shown with staining for SR-BI (i) and for S6 phosphorylation at serine 240 and 244 in a consecutive area of the same tumor (j). A representative low grade prostate cancer is shown with staining for SR-BI (k) and for S6 phosphorylation at serine 240 and 244 in a consecutive area of the same tumor (I). pS6 = ribosomal protein S6 phosphorylation at serine 240 and 244

Mechanistic studies have shown that mTOR signaling can mediate androgen independence [21]. Therefore, we further assessed the association of SR-BI expression with serine phosphorylation of ribosomal protein S6 at position 240 and 244. A total of 22 biopsy cores were subjected to immunohistochemical staining for pS6, and adjacent sections from the same patients were simultaneously subjected to SR-BI staining. Representative histologic staining for high grade and low grade prostate carcinoma samples is shown in Fig. 4 (i-l). After the pS6 and SR-BI staining, the samples were analyzed for their staining intensity. Representative cores for different staining intensities of $\mathrm{pS} 6$ and their respective scores are shown in Additional file 1: Figure S1 (A-D). Spearman correlation analysis revealed a significant positive correlation of SR-BI and pS6 $(\mathrm{R}=0.828, p<0.001)$.

\section{Discussion}

Prostate cancer is the most commonly diagnosed malignancy in men and it has the potential to progress to a metastatic and highly aggressive form of cancer, which is still difficult to cure. Therefore, it is of profound importance to identify markers that allow the prediction of prostate cancer progression to its aggressive metastatic form. Recent studies suggest that cholesterol plays a major role in prostate cancer [15, 22-24]. In human cells, cholesterol uptake is mainly based on two pathways: receptor-mediated endocytosis by the LDL receptor and selective lipid uptake by SR-BI [6-8]. Here, we show an association of prostate cancer malignancy with the expression of the HDL receptor SR-BI. Our analysis of 306 clinical prostate samples for mRNA and 106 prostate tissue biopsy cores for protein expression identified significantly higher SR-BI expression in high Gleason grade versus low Gleason grade prostate cancer samples. Furthermore, our analysis of gene expression profiles identified significantly higher SR-BI mRNA expression in metastatic compared to non-metastatic prostate cancer. Strikingly, we further discovered an association of SR-BI expression with disease-free survival time in a cohort of 85 clinical prostate samples. Previous studies already suggested a connection of SR-
BI expression with prostate cancer: the knockdown of SR-BI has been shown to reduce PSA levels and the viability of prostate cancer cells in vitro [15]. Moreover, SR-BI was found to be significantly up-regulated with progression to lethal castration-resistant prostate cancer (CRPC) in an LNCaP xenograft mouse model [25], while androgen-tolerant LNCaP cells in vitro did not show SR-BI up-regulation [26]. SR-BI has further been linked to nasopharyngeal cancer [27], colorectal cancer [28], ovarian cancer [28] and most notably breast cancer [29,30], a tumor strongly dependent on the synthesis of sexual hormones. Furthermore, it was demonstrated that mutations of SR-BI affected the proliferation and apoptosis of the breast cancer cell line MCF-7 [30]. Knockdown of SR-BI was shown to inhibit proliferation and migration in breast cancer, and SRBI knockdown also caused a decrease of tumor growth in MDA-MB231 and MCF-7 breast cancer cells in vivo when injected into nude mice [29].

The mTOR pathway plays a key role in the regulation of cellular growth and metabolism [31, 32]. Together with raptor and LST8, mTOR forms a complex called mTORC1 (mTOR complex 1), which acts by activating the ribosomal protein S6 through the protein kinase S6K1 [31, 32]. It is further known that mTORC1 influences cholesterol synthesis and uptake via the SREBP pathway [33-35]. Recently, it was shown that the inhibition of mTOR via rapamycin down-regulates SR-BI expression in human umbilical vein endothelial cells, indicating a direct connection between mTOR activation and SR-BI expression [36]. Further, it is known that mTOR plays a crucial role in the progression of prostate cancer to CRPC by influencing the androgen signaling pathway [37, 38]. According to our results, pS6 expression significantly correlates with SR-BI expression, which suggests the regulation of SR-BI by mTORC1 in prostate cancer.

To our knowledge, SR-BI has not been thoroughly studied in clinical samples of prostate cancer, and our findings on the mRNA and protein expression of SR-BI can contribute substantially to our understanding of prostate cancer progression. This study demonstrates the high expression of SR-BI in de-differentiated and metastasized 
prostate cancer, which almost always acquires resistance to androgen depletion. Therefore, we suggest that increased levels of SR-BI are involved in the transport of cholesterol into the tumor cell. This uptake of cholesterol could be exploited by the cancer cell to up-regulate its androgen synthesis. We observed the up-regulation of $3 \beta$ - and $17 \beta$-hydroxysteroid dehydrogenases, which may play an important yet unclear role in intra-tumoral androgen synthesis $[39,40]$. This process may contribute to the generation of castration-resistant prostate cancer. Therefore, pharmacologic inhibition of the HDL receptor might represent a way to inhibit prostate cancer progression. We suggest that SR-BI may be a valuable target for prostate cancer therapy; therefore, we strongly recommend that further studies investigate the role of SR-BI during prostate cancer progression.

\section{Conclusions}

Here we have shown that the HDL receptor SR-BI can be induced during the course of prostate cancer formation and progression. Intra-tumor expression was associated with an increase in Gleason scoring and also metastatic prostate tissue showed SR-BI up-regulation compared to primary tumor tissue. Importantly, we identified positive correlation of SR-BI expression with expression of androgen synthesizing enzymes and mTOR activation.

\section{Additional file}

Additional file 1: Figure S1. Immunohistochemical staining of prostate tissue for ribosomal protein phosphorylation at serine 240 and 244 . Each panel shows representative prostate samples scored for staining intensity as follows; 0 for negative (A), 1 for low (B), 2 for moderate (C) and 3 for high levels of pS6 (D). pS6 = ribosomal protein S6 phosphorylation at serine 240 and 244. (JPEG $537 \mathrm{~kb}$ )

\section{Competing interests}

The authors declare that they have no competing interests.

\section{Authors' contributions}

DS carried out the bioinformatic analysis, performed the statistical analysis and drafted the manuscript. KK carried out the immunohistologic analysis and drafted the manuscript. AP participated in immunohistologic staining and analysis. BS and CR carried out data analysis and interpretation. HS and $\mathrm{MH}$ participated in the design of the study and data interpretation. MM conceived of the study, participated in its design and coordination, and helped to draft the manuscript. All authors read and approved the final manuscript.

\section{Acknowledgements}

The authors are thankful for the excellent technical assistance of Jelena Brankovic.

This manuscript was edited for English language by American Journal Experts (AJE).

This work was supported by the Austrian Science Fund, FWF, grant number P25336-B13 (to Mario Mikula) and P25763-B13 (to Clemens Röhrl).

\section{Author details}

${ }^{1}$ Institute of Medical Genetics, Medical University of Vienna, Währinger Strasse 10, 1090 Vienna, Austria. ${ }^{2}$ Institute of Medical Chemistry, Medical University of Vienna, Währinger Strasse 10, 1090 Vienna, Austria.

Received: 11 May 2015 Accepted: 31 July 2015

Published online: 07 August 2015

\section{References}

1. Saraon P, Jarvi K, Diamandis EP. Molecular alterations during progression of prostate cancer to androgen independence. Clin Chem. 2011;57(10):1366-75. doi:10.1373/clinchem.2011.165977.

2. Haenszel W, Kurihara M. Studies of Japanese migrants. I. Mortality from cancer and other diseases among Japanese in the United States. J Natl Cancer Inst. 1968;40(1):43-68.

3. Shimizu H, Ross RK, Bernstein L, Yatani R, Henderson BE, Mack TM. Cancers of the prostate and breast among Japanese and white immigrants in Los Angeles County. Br J Cancer. 1991;63(6):963-6.

4. Bravi F, Scotti L, Bosetti C, Talamini R, Negri E, Montella M, et al. Self-reported history of hypercholesterolaemia and gallstones and the risk of prostate cancer. Ann Oncol. 2006;17(6):1014-7. doi:10.1093/annonc/mdl080.

5. Yue S, Li J, Lee SY, Lee HJ, Shao T, Song B, et al. Cholesteryl ester accumulation induced by PTEN loss and PI3K/AKT activation underlies human prostate cancer aggressiveness. Cell Metab. 2014;19(3):393-406. doi:10.1016/j.cmet.2014.01.019.

6. Acton S, Rigotti A, Landschulz KT, Xu S, Hobbs HH, Krieger M. Identification of scavenger receptor SR-BI as a high density lipoprotein receptor. Science. 1996;271(5248):518-20.

7. Krieger M. Charting the fate of the "good cholesterol": identification and characterization of the high-density lipoprotein receptor SR-BI. Annu Rev Biochem. 1999;68:523-58. doi:10.1146/annurev.biochem.68.1.523.

8. Rohrl C, Stangl H. HDL endocytosis and resecretion. Biochim Biophys Acta. 2013;1831(11):1626-33. doi:10.1016/j.bbalip.2013.07.014

9. Chen Y, Hughes-Fulford M. Human prostate cancer cells lack feedback regulation of low-density lipoprotein receptor and its regulator, SREBP2. Int J Cancer. 2001;91(1):41-5. doi:10.1002/10970215(20010101)91:1<41::AID-IJC1009>3.0.CO;2-2.

10. Connelly MA. SR-BI-mediated HDL, cholesteryl ester delivery in the adrenal gland. Mol Cell Endocrinol. 2009:300(1-2):83-8. doi:10.1016/.mce.2008.09.011.

11. Temel RE, Trigatti B, DeMattos RB, Azhar S, Krieger M, Williams DL. Scavenger receptor class $B$, type I (SR-BI) is the major route for the delivery of high density lipoprotein cholesterol to the steroidogenic pathway in cultured mouse adrenocortical cells. Proc Natl Acad Sci U S A. 1997;94(25):13600-5

12. Azhar S, Leers-Sucheta S, Reaven E. Cholesterol uptake in adrenal and gonadal tissues: the SR-BI and 'selective' pathway connection. Front Biosci. 2003:8:5998-1029.

13. Fruhwurth S, Kovacs WJ, Bittman R, Messner S, Rohrl C, Stangl H. Differential basolateral-apical distribution of scavenger receptor, class B, type I in cultured cells and the liver. Histochem Cell Biol. 2014. doi:10.1007/s00418-014-1251-9.

14. Vergeer M, Korporaal SJ, Franssen R, Meurs I, Out R, Hovingh GK, et al. Genetic variant of the scavenger receptor BI in humans. N Engl J Med. 2011;364(2):136-45. doi:10.1056/NEJMoa0907687.

15. Twiddy AL, Cox ME, Wasan KM. Knockdown of scavenger receptor class $B$ type I reduces prostate specific antigen secretion and viability of prostate cancer cells. Prostate. 2012;72(9):955-65. doi:10.1002/pros.21499.

16. Lapointe J, Li C, Higgins JP, van de Rijn M, Bair E, Montgomery K, et al. Gene expression profiling identifies clinically relevant subtypes of prostate cancer. Proc Natl Acad Sci U S A. 2004;101(3):811-6. doi:10.1073/pnas.0304146101.

17. Grasso CS, Wu YM, Robinson DR, Cao X, Dhanasekaran SM, Khan AP, et al. The mutational landscape of lethal castration-resistant prostate cancer. Nature. 2012;487(7406):239-43. doi:10.1038/nature11125.

18. Yu YP, Landsittel D, Jing L, Nelson J, Ren B, Liu L, et al. Gene expression alterations in prostate cancer predicting tumor aggression and preceding development of malignancy. J Clin Oncol. 2004;22(14):2790-9. doi:10.1200/JCO.2004.05.158.

19. Chandran UR, Ma C, Dhir R, Bisceglia M, Lyons-Weiler M, Liang W, et al, Gene expression profiles of prostate cancer reveal involvement of multiple molecular pathways in the metastatic process. BMC Cancer. 2007;7:64. doi:10.1186/1471-2407-7-64. 
20. Gulzar ZG, McKenney JK, Brooks JD. Increased expression of NuSAP in recurrent prostate cancer is mediated by E2F1. Oncogene. 2013;32(1):70-7. doi:10.1038/onc.2012.27.

21. Inoue T, Yoshida T, Shimizu Y, Kobayashi T, Yamasaki T, Toda Y, et al. Requirement of androgen-dependent activation of protein kinase Czeta for androgen-dependent cell proliferation in LNCaP Cells and its roles in transition to androgen-independent cells. Mol Endocrinol. 2006;20(12):3053-69. doi:10.1210/me.2006-0033.

22. Llaverias G, Danilo C, Wang Y, Witkiewicz AK, Daumer K, Lisanti MP, et al. A Western-type diet accelerates tumor progression in an autochthonous mouse model of prostate cancer. Am J Pathol. 2010;177(6):3180-91. doi:10.2353/ajpath.2010.100568.

23. Sekine Y, Demosky SJ, Stonik JA, Furuya Y, Koike H, Suzuki K, et al. High-density lipoprotein induces proliferation and migration of human prostate androgenindependent cancer cells by an ABCA1-dependent mechanism. Mol Cancer Res. 2010;8(9):1284-94. doi:10.1158/1541-7786.MCR-10-0008.

24. Thysell E, Surowiec I, Hornberg E, Crnalic S, Widmark A, Johansson Al, et al. Metabolomic characterization of human prostate cancer bone metastases reveals increased levels of cholesterol. PLoS ONE. 2010;5(12):e14175. doi:10.1371/journal.pone.0014175.

25. Leon CG, Locke JA, Adomat HH, Etinger SL, Twiddy AL, Neumann RD, et al. Alterations in cholesterol regulation contribute to the production of intratumoral androgens during progression to castration-resistant prostate cancer in a mouse xenograft model. Prostate. 2010;70(4):390-400. doi:10.1002/pros.21072.

26. Krycer JR, Brown AJ. Does changing androgen receptor status during prostate cancer development impact upon cholesterol homeostasis? PLoS ONE. 2013;8(1):e54007. doi:10.1371/journal.pone.0054007.

27. Zheng Y, Liu Y, Jin H, Pan S, Qian Y, Huang C, et al. Scavenger receptor B1 is a potential biomarker of human nasopharyngeal carcinoma and its growth is inhibited by HDL-mimetic nanoparticles. Theranostics. 2013;3(7):477-86. doi:10.7150/thno.6617.

28. Shahzad MM, Mangala LS, Han HD, Lu C, Bottsford-Miller J, Nishimura M, et al. Targeted delivery of small interfering RNA using reconstituted high-density lipoprotein nanoparticles. Neoplasia. 2011;13(4):309-19.

29. Danilo C, Gutierrez-Pajares JL, Mainieri MA, Mercier I, Lisanti MP, Frank PG. Scavenger receptor class B type I regulates cellular cholesterol metabolism and cell signaling associated with breast cancer development. Breast Cancer Res. 2013;15(5):R87. doi:10.1186/bcr3483.

30. Cao WM, Murao K, Imachi H, Yu X, Abe H, Yamauchi A, et al. A mutant high-density lipoprotein receptor inhibits proliferation of human breast cancer cells. Cancer Res. 2004;64(4):1515-21.

31. Laplante M, Sabatini DM. mTOR signaling in growth control and disease. Cell. 2012;149(2):274-93. doi:10.1016/j.cell.2012.03.017.

32. Ma XM, Blenis J. Molecular mechanisms of mTOR-mediated translationa control. Nat Rev Mol Cell Biol. 2009;10(5):307-18. doi:10.1038/nrm2672.

33. Lewis CA, Griffiths B, Santos CR, Pende M, Schulze A. Regulation of the SREBP transcription factors by mTORC1. Biochem Soc Trans. 2011;39(2):495-9. doi:10.1042/BST0390495

34. Laplante M, Sabatini DM. An emerging role of mTOR in lipid biosynthesis. Curr Biol. 2009:19(22):R1046-52. doi:10.1016/j.cub.2009.09.058.

35. Preitschopf A, Li K, Schorghofer D, Kinslechner K, Schutz B, Thi Thanh Pham H, et al. mTORC1 is essential for early steps during Schwann cell differentiation of amniotic fluid stem cells and regulates lipogenic gene expression. PLoS ONE. 2014;9(9):e107004. doi:10.1371/journal.pone.0107004.

36. Fruhwurth S, Krieger S, Winter K, Rosner M, Mikula M, Weichhart T, et al. Inhibition of mTOR down-regulates scavenger receptor, class B, type I (SR-BI) expression, reduces endothelial cell migration and impairs nitric oxide production. Biochim Biophys Acta. 2014;1841(7):944-53. doi:10.1016/j.bbalip.2014.03.014

37. Bitting RL, Armstrong AJ. Targeting the PI3K/Akt/mTOR pathway in castration-resistant prostate cancer. Endocr Relat Cancer. 2013;20(3):R83-99. doi:10.1530/ERC-12-0394

38. Edlind MP, Hsieh AC. PI3K-AKT-mTOR signaling in prostate cancer progression and androgen deprivation therapy resistance. Asian J Androl. 2014;16(3):378-86. doi:10.4103/1008-682X.122876.

39. Labrie F, Luu-The V, Lin SX, Labrie C, Simard J, Breton R, et al. The key role of 17 beta-hydroxysteroid dehydrogenases in sex steroid biology. Steroids. 1997;62(1):148-58

40. Luu-The V, Labrie C, Simard J, Lachance Y, Zhao HF, Couet J, et al. Structure of two in tandem human 17 beta-hydroxysteroid dehydrogenase genes. Mol Endocrinol. 1990;4(2):268-75. doi:10.1210/mend-4-2-268.

\section{Submit your next manuscript to BioMed Central and take full advantage of:}

- Convenient online submission

- Thorough peer review

- No space constraints or color figure charges

- Immediate publication on acceptance

- Inclusion in PubMed, CAS, Scopus and Google Scholar

- Research which is freely available for redistribution

Submit your manuscript at www.biomedcentral.com/submit 